\title{
STATICS, STRUCTURE, AND RESIDUAL NORMAL MOVEOUT: EXACT MATHEMATICAL SOLUTIONS IN SIMPLE FORM*
}

\author{
BY \\ I. LERCHE \\ University of South Carolina
}

\begin{abstract}
The separation of seismic reflection times into the component parts due to source static, receiver static, structure time, and residual normal moveout is exhibited in terms of simple differential and integral operations on the reflection time. The solution is valid for all component parts which possess an $L_{2}$ norm on the open one-dimensional domain of common midpoint. The equations yield simple, explicit solutions for each separate component. Possible methods of implementing these direct solutions for real travel-time data are presented with suggestions for avoiding, or bypassing, the differential operations. These solutions are presented both to provide a novel viewpoint for examining the so-called statics equation and as an aid to those who are actively pursuing the problem of finding methods for successfully optimizing statics problems in divers and diverse situations.
\end{abstract}

1. Introduction. The problem of obtaining expressions for receiver static, source static, residual normal moveout, and structural terms given the travel time $T(x, y)$ as a function of the common midpoint (CMP) $x$ and offset $y$ has conventionally been attacked by using (a) expansions in terms of orthogonal functions followed by a least-squares approach to determine the best values of the expansion coefficients [3], (b) trigonometric expansion again followed by a least-squares approach to obtain the coefficients of the trigonometric expansion [1], or (c) direct spectral inversion for each desired wavelength [2]. The purpose of the present paper is to point out that the conventional statics equation, regarded explicitly as a mathematical equation in its own right, has an exact unique solution for the receiver, source, residual normal moveout (RNMO), and structure terms provided only that each of them has a bounded Fourier transform (of measure $L_{2}$ on the open set). The solution is exhibited in terms of integrations and differentiations applied to the travel time $T(x, y)$. Armed with this exact solution, the numerical accuracy of the travel-time data then becomes the overriding concern for determining the precision of the four terms as functions of CMP. The improvement is clear: the problem of "solving" the statics equation is no longer tied so tightly to the accuracy of the travel-time data.

\footnotetext{
* Received May 22, 1985.
} 
Concentrated efforts to improve the travel-time data so that the relevant statics factors (for which exact expressions are now available) are most sharply defined, and to provide a "best" method for implementing the mathematical solution in accord with given data quality, now become the major goals, in line with the philosophy of Wiggins et al. [3] and Marcoux [2].

Section 2 sets up and solves the statics equation in terms of mathematical operations on the travel time regarded as a smoothly continuous function of CMP and offset. The solution clearly separates the terms due to source, receiver, RNMO, and structure.

Section 3 suggests some ways in which the expressions for the four terms can be determined from real data and also suggests ways of encoding the solution presented so that we can obtain the best practical method of implementing the mathematical solution, and compare and contrast the accuracy of this solution with the conventional methods of handling statics problems. Our concern in this paper is not so much with the overall problem of providing general methods of attack for optimally delineating all successful statics applications. With Marcoux [2], we recognize that such would be the subject of a very long paper indeed. Instead, our major thrust is to attempt to express directly, in terms of simple differentiations and integrations applied to the travel time, the receiver statics, source statics, RNMO correction, and structure term viewed as a mathematical equation. Methods for handling the imprecision of real data pertaining to the statics equation have been entered into by others (e.g., [2, 3]).

Our hope is that by presenting a different method of viewing the statics equation we will stimulate research into more and better ways of allowing for data imprecision in real situations.

2. The statics equation and its solution. The computation of residual "statics" corrections to seismic waves takes place in two steps. First is the measurement of reflection times by some method such as cross-correlation of each seismic trace with a reference trace. The second stage, and the one we are concerned with here, is the reduction of the measured travel times according to some geophysical model, including structure and (RNMO) terms as well as surface consistent statics.

A simple illustration of the static component of model reflection times as a function of (CMP) and offset is shown in Fig. 1 (taken from Marcoux [2]), where the source static appears as a positive dipping event, whereas the receiver static shows negative dip.

Conventionally [1-3], the travel time $T(x, y)$ is taken to satisfy the equation

$$
T(x, y)=R(x+y)+S(x-y)+y^{2} M(x)+C(x),
$$

where

$$
\begin{aligned}
R(x+y) & =\text { receiver statics term } \\
S(x-y) & =\text { source statics term } \\
M(x) y^{2} & =\text { RNMO correction } \\
C(x) & =\text { structure term } \\
x & =\mathrm{CMP} \\
y & =\text { offset } / 2 .
\end{aligned}
$$




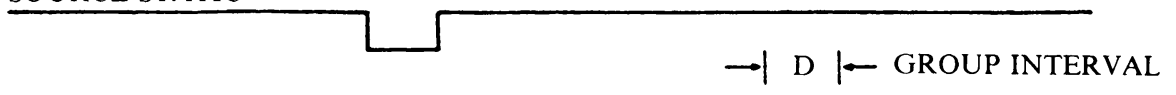

(Offset/Trace)

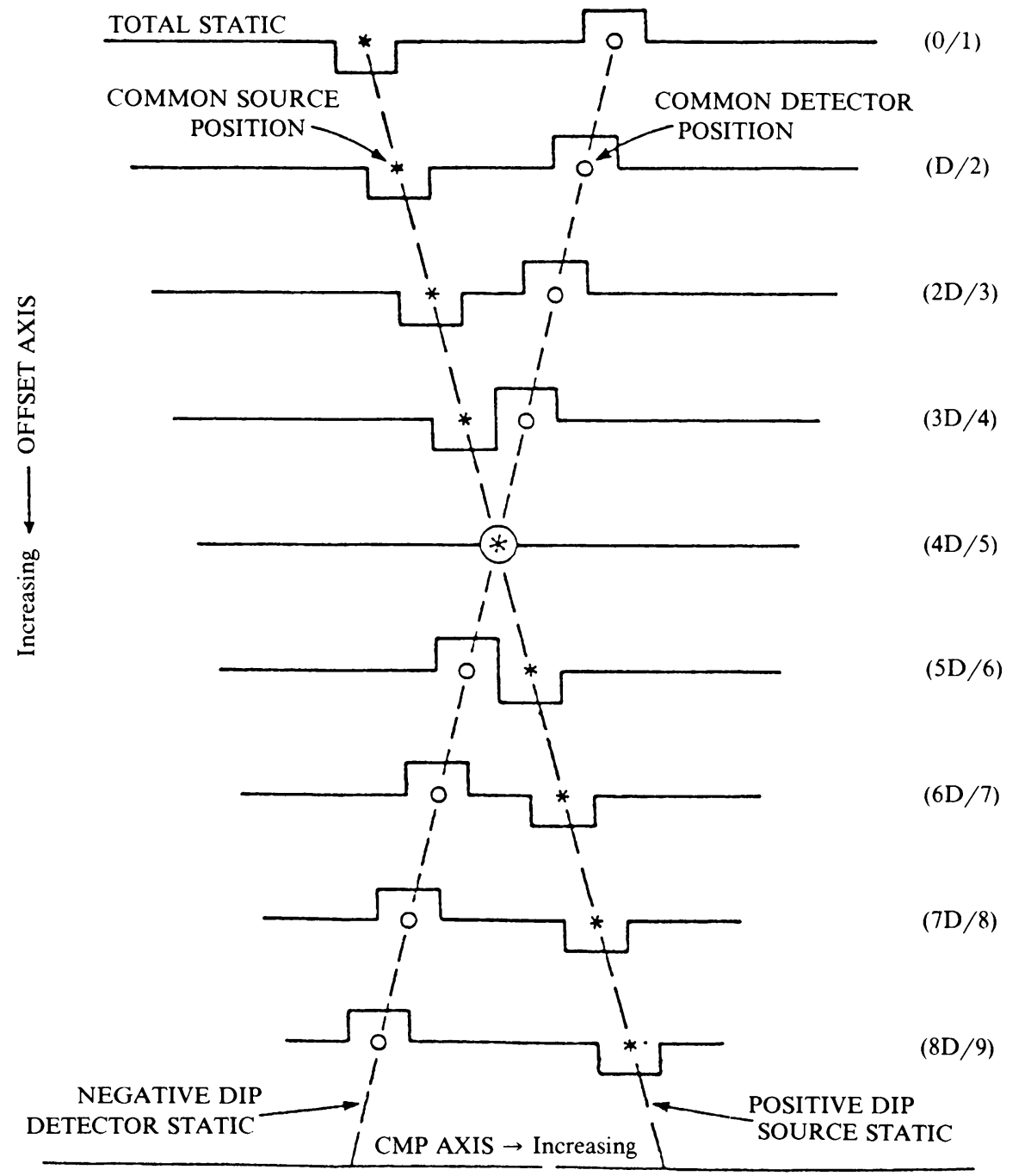

FIG. 1. Pictorial representation of the static component of model reflection times as a function of common midpoint (CMP) and offset. The source static appears as a positive dipping event, whereas the receiver static shows negative dip. (From Marcoux [2].)

The question is, does Eq. (1) have a unique solution in which the terms $R(x), S(x)$, $M(x)$, and $C(x)$ can be individually obtained from the travel time $T(x, y)$ and, if so, how is the solution expressed in terms of simple differential and integral manipulations on the travel time? We will show here that the answer is yes under the mathematical condition 
that $T(x, y)$ is known and bounded for all $x$ and $y$. As a reviewer of this paper correctly noted, it is important also to point out that the computation of the unknown functions at a given $(x, y)$ pair requires measurement of $T(x, y)$ only up to some bounding $(x, y)$. We shall return to this point later in Sec. 3, where we shall also consider the limitations imposed by finite range, and discrete, data for $T(x, y)$.

In order to solve Eq. (1) exactly, we Fourier transform w.r.t. $x$ so that

$$
\begin{aligned}
\tau(k, y) & =\int_{-\infty}^{\infty} T(x, y) \exp (-i k x) d x, \\
{[m(k), c(k), r(k), s(k)] } & =\int_{-\infty}^{\infty}[M(x), C(x), R(x), S(x)] \exp (-i k x) d x .
\end{aligned}
$$

(Note the fundamental assumption that these Fourier transforms exist and are bounded. This eliminates ab initio any nonuniqueness resulting from unbounded functions-such as the arbitrary linear solutions reported by Wiggins et al. [3].)

The Fourier-transformed version of Eq. (1) is

$$
\tau(k, y)=r(k) e^{i k y}+s(k) e^{-i k y}+y^{2} m(k)+c(k) .
$$

But equation (3) must be valid for all $y$. Hence differentiate equation (3) three times with respect to $y$ to obtain

$$
\begin{gathered}
\frac{\partial \tau(k, y)}{\partial y}=i k\left[r(k) e^{i k y}-s(k) e^{-i k y}\right]+2 y m(k), \\
\frac{\partial^{2} \tau(k, y)}{\partial y^{2}}=-k^{2}\left[r(k) e^{i k y}+s(k) e^{-i k y}\right]+2 m(k),
\end{gathered}
$$

and

$$
\frac{\partial^{3} \tau(k, y)}{\partial y^{3}}=-i k^{3}\left[r(k) e^{i k y}-s(k) e^{-i k y}\right] .
$$

Equations (3) through (6) are four linear algebraic equations for the four unknowns $r(k)$, $m(k), s(k)$, and $c(k)$. They can be solved exactly to give

$$
\begin{aligned}
& m(k)=(2 y)^{-1}\left[\frac{\partial \tau}{\partial y}+k^{-2} \frac{\partial^{3} \tau}{\partial y^{3}}\right], \\
& c(k)=\tau-\left[\frac{1}{2} y+\left(k^{2} y\right)^{-1}\right] \frac{\partial \tau}{\partial y}+k^{-2} \frac{\partial^{2} \tau}{\partial y^{2}}-k^{-2}\left[\frac{1}{2} y+\left(y k^{2}\right)^{-1}\right] \frac{\partial^{3} \tau}{\partial y^{3}}, \\
& r(k)=\left(2 k^{2}\right)^{-1} e^{-i k y}\left[y^{-1} \frac{\partial \tau}{\partial y}-\frac{\partial^{2} \tau}{\partial y^{2}}+\left(i k^{-1}+\left(k^{2} y\right)^{-1}\right) \frac{\partial^{3} \tau}{\partial y^{3}}\right] \\
& s(k)=\left(2 k^{2}\right)^{-1} e^{i k y}\left[y^{-1} \frac{\partial \tau}{\partial y}-\frac{\partial^{2} \tau}{\partial y^{2}}+\left(-i k^{-1}+\left(k^{2} y\right)^{-1}\right) \frac{\partial^{3} \tau}{\partial y^{3}}\right] .
\end{aligned}
$$

Inverting the Fourier transforms by using equations (7) through (10) then provides expressions for the four terms $R(x), S(x), M(x)$, and $C(x)$ in terms of simple differential and integral operations on the travel time. With the aid of the auxiliary functions

$$
I_{1}(x, y)=\int_{0}^{x} T\left(x^{\prime}, y\right) d x^{\prime},
$$




$$
I_{n+1}(x, y)=\int_{0}^{x} I_{n}\left(x^{\prime}, y\right) d x^{\prime}, \quad n \geqslant 1
$$

we have

$$
\begin{aligned}
M(x)= & (2 y)^{-1}\left[\frac{\partial T(x, y)}{\partial y}-\frac{\partial^{3} I_{2}(x, y)}{\partial y^{3}}\right] \\
C(x)= & T(x, y)-\frac{1}{2} y \frac{\partial T(x, y)}{\partial y}+y^{-1} \frac{\partial I_{2}(x, y)}{\partial y}-\frac{\partial^{2} I_{2}(x, y)}{\partial y^{2}} \\
& -y^{-1} \frac{\partial^{3} I_{4}(x, y)}{\partial y^{3}}+\frac{1}{2} y \frac{\partial^{3} I_{2}(x, y)}{\partial y^{3}}, \\
R(x)= & T(x-y, y)+y^{-1} \frac{\partial I_{2}(x-y, y)}{\partial y}+\frac{5}{2} \frac{\partial I_{1}(x-y, y)}{\partial y} \\
& +2 \frac{\partial^{2} I_{2}(x-y, y)}{\partial y^{2}}+\frac{3}{2} y^{-1} \frac{\partial^{2} I_{3}(x-y, y)}{\partial y^{2}} \\
& +\frac{1}{2} y^{-1} \frac{\partial^{3} I_{4}(x-y, y)}{\partial y^{3}}+\frac{1}{2} \frac{\partial^{2} I_{3}(x-y, y)}{\partial y^{3}}
\end{aligned}
$$

and

$$
\begin{aligned}
S(x)= & T(x+y, y)+y^{-1} \frac{\partial I_{2}(x+y, y)}{\partial y}-\frac{5}{2} \frac{\partial I_{1}(x+y, y)}{\partial y} \\
& +2 \frac{\partial^{2} I_{2}(x+y, y)}{\partial y^{2}}-\frac{3}{2} y^{-1} \frac{\partial^{2} I_{3}(x+y, y)}{\partial y^{2}} \\
& +\frac{1}{2} y^{-1} \frac{\partial^{3} I_{4}(x+y, y)}{\partial y^{3}}-\frac{1}{2} \frac{\partial^{3} I_{3}(x+y, y)}{\partial y^{3}} .
\end{aligned}
$$

Equations (12) through (15) represent the unique solution to the problem of separating terms due to the source, receiver, RNMO, and structure on the conventional assumption that the travel time can indeed be represented by equation (1).

Several points are relevant. This seems to be the first time that an exact solution has been found for the statics equation (1) in terms of simple operations on the travel time. Second, the terms $M(x), C(x), R(x)$, and $S(x)$ are, by definition, independent of the offset $y$ and are supposedly dependent only on the common midpoint variable $x$. The right-hand sides of equations (12) through (15), however, do not appear to provide expressions which are manifestly independent of the offset $y$. This is coupled to the fact that if, and only if, the travel time satisfies Eq. (1) will the receiver, source, RNMO, and structure static terms be functions solely of CMP. Any departure of the travel time from the assumed form of Eq. (1) will lead to offset dependences for the four terms. This is not unlike the problem of attempting to find $m$ and $c$ consistent with $y=m x+c$. There we know that

$$
m=d y / d x, \quad c=y-m x \equiv y-x d y / d x .
$$

Clearly, only if $y$ is indeed linear in $x$ will $m$ and $c$ be constants. But for a given data set $y(x)$ we often ask for the constant values of $m$ and $c$ which can "best" satisfy the data. 
Here for a finite data set of $T(x, y)$ the analogy is that we want to search for the "best" values of $R, S, M$, and $C$ which satisfy the given data set on $T(x, y)$.

There is the further point that in real data situations information on travel time is over a restricted range of offsets and CMPs-contrary to the requirements of the solution method, where Fourier transforms and their inverses are performed as a matter of course as though such information is available. Thus the pragmatic determination of $C(x)$, $M(x), R(x)$, and $S(x)$ will include errors produced by this lack of detailed knowledge.

Perhaps the greatest error in determining the four individual corrections arises from the differentiations of the travel time with respect to offset (or of integrals of travel time with respect to CMP). While the integrations with respect to CMP will tend to smooth out vagaries in data, differentiation will tend to enhance discordances.

In the next section we address briefly some of these pragmatic considerations for the functional form of solution we provide, but recognizing that a considerable amount of research still needs to be done to improve on our primitive exposition.

3. Implementation of the statics solution. Since the exact four terms $R(x), S(x), M(x)$, and $C(x)$ are nominally independent of offset we can use this fact to try to obtain, from limited data, approximations to the true statics terms by using variants of the exact solutions given by equations (12) through (15).

For instance, we see that the expressions for $R, S, M$, and $C$ all involve derivatives up to third order with respect to offset. Consider then a set of $N$ measurements of the travel time $T_{i}(i=2, \ldots, N)$ made at a set of increasing offsets $y_{i}(i=1,2, \ldots, N)$. For each set of four measurements $T_{i-1}, T_{i}, T_{i+1}$, and $T_{i+2}$ at the offsets $y_{i-1}, y_{i}, y_{i+1}$, and $y_{i+2}$ one can construct approximations to the derivatives in equations (12) through (15). Thus each sequential set of four measurements provides the estimate $R_{i}, S_{i}, M_{i}$, and $C_{i}$ of the four statics terms. By superposing these estimates and sequentially increasing $i$ one can construct a "smooth" estimate of each of the four statics terms which is not so beholden to any one set of four individual measurements, ${ }^{1}$ any of which contains some measurement error.

Alternatively, one can see the extent to which an individual estimate of the statics terms departs significantly from the average value and discard those estimates exceeding some preassigned criteria of uniformity since the statics terms are nominally independent of offset - if the travel time does indeed separate as in Eq. (1).

An estimate of the degree of mismatch between the exact analytic solution to the statics equation and measurements of the travel time can be obtained by taking the smooth numerical estimates of $R, S, M$, and $C$ and constructing

$$
Z^{2} \equiv N^{-1} \sum_{i=1}^{N}\left[T\left(x, y_{i}\right)-R\left(x+y_{i}\right)-S\left(x-y_{i}\right)-y_{i}^{3} M(x)-C(x)\right]^{2} \geqslant 0 .
$$

The variation of $Z^{2}$ with $x$ provides an indication of the domains where the data are sufficiently accurate that a good residual statics solution has been obtained. The RMS residual time correction with CMP, $x$, is then just $Z(x)$.

\footnotetext{
${ }^{1}$ This is also the reason that we did not use just four measurements of $\tau(k, y)$ in Eq. (3) to determine $r(k)$, $s(k), m(k)$, and $c(k)$.
} 
A third possibility is to note that by multiplying the exact solutions by powers of $y$ and then integrating over offset, moment equations can be generated which obviate the differentiations and their attendant potential for error magnification.

For instance, from Eq. (12) we see that multiplying by $y^{p+1}$ and integrating over $Y_{\min } \leqslant y \leqslant Y_{\max }$ enables us to write

$$
\begin{aligned}
M(x)(p+2)^{-1}\left[Y_{\max }^{p+2}-Y_{\min }^{p+2}\right] & =\frac{1}{2}\left[Y_{\max }^{P} T\left(x, Y_{\max }\right)-Y_{\min }^{P} T\left(x, Y_{\min }\right)\right. \\
& \left.-p \int_{Y_{\min }}^{Y_{\max }} y^{p-1} T(x, y) d y-\int_{Y_{\min }}^{Y_{\max }} y^{p-1} \frac{\partial^{3} I_{2}(x, y)}{\partial y^{3}} d y\right] .
\end{aligned}
$$

The last term in Eq. (17) can be integrated three times by parts so that only the first and second derivatives of $I_{2}$ at the end points are needed rather than throughout the whole dynamic range of offset.

Similar moments can be taken of the other statics terms to alleviate substantially the practical problems of constructing functional derivatives from discrete, bounded, and noisy travel-time data. The questions of (i) what range of offsets are "best" to use and (ii) the relevant powers $p$ they should be raised to in attempting to obtain the normally offset independent factors $M(x), R(x), C(x)$, and $S(x)$ are problems we do not address here. But one thought is that by minimizing $Z^{2}\left(p, Y_{\min }, Y_{\max }\right)$, the degree of mismatch, with respect to choices for $p, Y_{\min }$, and $Y_{\max }$ one can determine for a given data set the best range of values to use.

4. Discussion and conclusion. The major point being made in this paper is that the conventional statics equation does have a unique solution in which the terms due to receiver statics, source statics, residual normal moveout, and structure are expressible directly in terms of the travel time, its integrals with respect to CMP, and its derivatives with respect to offset, provided only that appropriate Fourier transforms exist on the open set.

This is apparently the first time that such a solution has been given in terms of simple differentiations and integrations applied to travel time.

Armed with this solution, the problem of constructing the four terms from real data is moved to a different level of difficulty. The conventional techniques of attempting to fit to the statics terms by orthogonal expansions, trigonometric series, or direct spectral inversion now are more directly tied to the problem of seeing how well real travel-time data can be made to fit the exact solution to the statics equation. The problems of numerically representing the exact analytic solution and of allowing for the inexactitudes of real travel-time data are the major remaining concerns.

\section{REFERENCES}

[1] F. Kirchheimer, Long period static analysis by trigonometric approximation, Paper S6.1, presented at the S.E.G. Meeting, Las Vegas, Nevada (September 14, 1983)

[2] M. O. Marcoux, On the resolution of statics, structure and residual normal movement, Geophysics 46, 984-993 (1981)

[3] R. A. Wiggins, K. L. Larner. R. C. Wisecup. Residual static analysis as a general linear inverse problem. Geophysics 41, 922-938 (1976) 